\title{
A Novel Cross Coding Scheme for OFDM Systems
}

\author{
Xiaoying Shao, Cornelis H. Slump \\ x.shao@ewi.utwente.nl, c.h.slump@ewi.utwente.nl \\ University of Twente, Faculty of EEMCS, Signals and Systems Group \\ P.O. box 217 - 7500 AE Enschede - The Netherlands
}

\begin{abstract}
In wireless OFDM-based systems, coding jointly over all the sub-carriers simultaneously performs better than coding separately per sub-carrier. However, the joint coding is not always optimal. In this paper, we propose a novel coding scheme based on fountain codes, which combines the separate coding and the joint coding over all the sub-carriers. The key element in the new proposed system is that each fountain-encoded packet is transmitted over a single sub-carrier. The packets can be discarded if they have encountered a low-energy channel. Fountain codes can recover the source data by using only the surviving packets. With this new approach, we have a gain of around $8.5 \mathrm{~dB}$ comparing to the FEC layer used in current WLAN standards (i.e. the IEEE 802.11a standard and the IEEE 802.11n standard).
\end{abstract}

\section{INTRODUCTION}

It is a challenge to communicate both reliably and at a high throughput, because the wireless channel is a hostile environment which suffers from time-varying multi-path propagation and high levels of man-made interference. To overcome the multi-path effect, Orthogonal Frequency Division Multiplexing (OFDM) has become a popular scheme for recent wireless systems which operate at a high bit rate [1]. For the effects of the noise and interference encountered in the transmission of the signal through the wireless channel, Error Correction Coding is used as a means of utilizing wireless channels at full capacity [2].

In [3], the authors mention that over a finite block length, coding jointly over the sub-carriers yields a smaller error probability than can be achieved by coding separately over the sub-carriers at the same rate. This theory has been applied in practical OFDM-based wireless systems. In the current Forward Error Correction (FEC) layer for OFDM systems (e.g. WLAN, DVB, DAB, etc), the source bits are encoded jointly across the sub-carriers. However, coding can also be done in a crosswise way, which combines the separate coding and the joint coding over all the sub-carriers. It is unknown whether the cross coding approach performs better or worse than the joint coding scheme in OFDM systems. Hence, it is of interest to investigate the performance of the cross coding scheme for OFDM systems.

In this paper, we propose a novel cross FEC layer based on fountain codes, which is called Opportunistic Error Correction Layer. In [4], MacKay describes the encoder of a fountain code as a metaphorical fountain that produces a stream of encoded packets. Anyone who wishes to receive the encoded file holds a bucket under the fountain and collects enough packets to recover the original data. It does not matter which packets are received, only a minimum amount of packets have to be received correctly [4]. In other words, fountain-encoded packets are independent with respect to each other.

To apply fountain codes in the OFDM system, we divide a block of source bits into a set of packets, which are encoded by a fountain code. Because fountain codes are only designed for erasure channels, we have to apply error correction codes to convert the wireless channel into an erasure channel. A fountain-encoded packet is transmitted over a subcarrier. Thus, multiple packets are transmitted simultaneously, using frequency division multiplexing. The receiver discards fountain-encoded packets which are transmitted over the subcarriers with deep fading. In other words, the receiver does not have to process all the received packets but only the packets from high-energy channels. The processing power is reduced correspondingly.

There are two coding steps in the opportunistic error correction layer. First, the source packets are coded jointly over all the sub-carriers by fountain codes; then, each fountainencoded packet is encoded individually over a sub-carrier by error correction codes. This is different from the traditional FEC layer. In this paper, we investigate the performance of this new cross coding scheme for the wireless systems.

The outline of this paper is as follows. First, the different coding schemes for wireless channels are discussed; this is followed by the opportunistic error correction layer. In Section IV, a description is given of the IEEE 802.11a system, which is a practical example of an OFDM system. We compare the current FEC layers with the opportunistic error correction layer in the simulation. Finally, the simulation results are analyzed. The paper ends with a discussion of the results.

\section{Coding FOR Wireless Channels}

The wireless channel is a hostile environment and often modeled as a frequency selective channel. The multi-path effect results in Inter-Symbol Interference (ISI). OFDM has become a fruitful approach to communicate over frequency selective channels [3]. In the OFDM system, each sub-carrier can be considered as a flat fading channel. However, due to the channel noise or interference, reliable communication still can not be guaranteed by only using OFDM.

Error correction codes have been applied in wireless systems for reliable communications. In OFDM systems, coding is performed in the frequency domain. Whether the source data is encoded jointly or separately over all the sub-carriers depends 


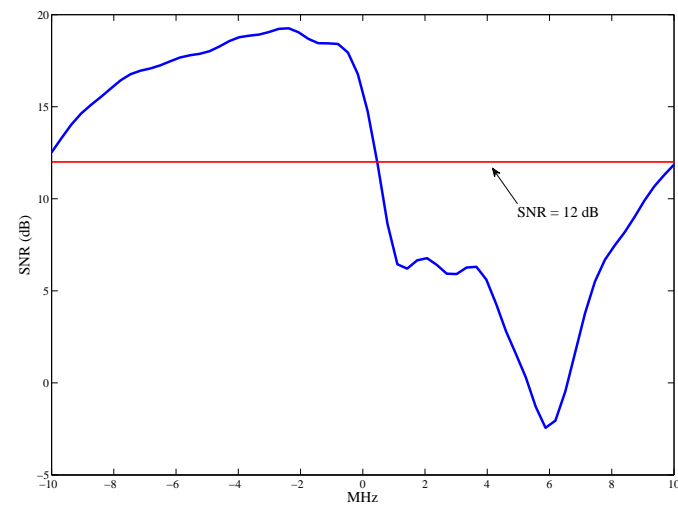

Fig. 1. Example of the baseband transfer function of a frequency selective Channel model A [5].

on the transmission mode. There are two modes to transmit an encoded packet:

- Mode I is to transmit each encoded packet over one subcarrier. In such case, the coding is performed separately over all the sub-carriers.

- Mode II is to transmit each encoded packet over all the sub-carriers like current WLAN systems. With this method, the coding is done jointly over all the subcarriers.

Indeed, over a finite block length, coding jointly over all the sub-carriers yields a smaller error probability than can be achieved by coding separately at the same rate [3]. That is because the sub-carrier with high energy can compensate for the sub-carrier with deep fading. This is also shown in the simulation results of Section $\mathrm{V}$.

From the power consumption point of view, however, the joint coding over all the sub-carriers is not optimal. With the joint coding, the receiver has to process all the received packet as it is not beforehand known from the channel knowledge, whether a packet is decodable or not. Take Figure 1 as an example, 1000 packets are transmitted over this channel. Each packet has 175 bits and encoded by a $(175,255)$ LDPC code [6]. QAM-16 is used as the mapping scheme and the channel estimation is assumed to be perfect. With the transmission Mode I, the receiver has to process all the 1000 packets and only 17 packets can be decoded correctly. With the transmission Mode II, the receiver can only process "good" packets which are transmitted over the sub-carriers with high energy, since each sub-carrier can be considered as a flat fading channel. For the LDPC code used in this example, it has a BER of $10^{-5}$ or lower when $\mathrm{SNR} \geq 12 \mathrm{~dB}$. In such case, the receiver only need to decode 500 packets in this example, which can all be decoded correctly in this example. Therefore, Mode II has lower processing power than Mode I. In addition, Mode II has less packets in error than Mode I in some "bad" channels like Figure 1.

Low power consumption in battery-powered wireless receivers is highly desirable. In order to lower the processing power with a low BER, we propose a novel cross coding approach based on fountain codes (i.e. opportunistic error correction layer) for wireless OFDM-based systems. The opportunistic error correction layer is based on Mode II but it is not a separate coding scheme. It is a combination of the separate coding and the joint coding over all the sub-carriers. We will discuss this new coding scheme in the next section.

\section{OpPORTUNISTIC ERROR CORRECTION}

The opportunistic error correction layer is based on fountain codes. In this paper, we use a kind of fountain codes, i.e. Luby Transform (LT) codes [7] in the proposed error correction layer. Other fountain codes (e.g. Raptor codes [8]) can also be applied.

Consider a block of size $K$ source packets $s_{1}, s_{2}, \cdots, s_{K}$ to be encoded by a fountain code. A "packet" has $m$ bits and is considered as a unity. At each clock cycle, labeled by $t$, one fountain-encoded packet is generated by selecting a set of source packets randomly and computing the bitwise sum (XOR) of these source packets [4]. The fountain codes can supply unlimited packets. In practical systems, only a fixed number of packets $N_{t}$ is generated.

At the receiver side, a certain amount of packets is required for successful decoding. The required number of received packets $N$ is slightly larger than the number of source packets $K$ and defined as:

$$
N=K(1+\varepsilon)
$$

where $\varepsilon$ is the percentage of extra packets and is called the overhead.

After receiving $N$ packets, the receiver can recover the source packets by the message-passing algorithm [9] which has a linear decoding cost. By using the message-passing algorithm, the practical block size of LT codes with small overhead $\varepsilon$ (i.e. within $5 \%$ ) is on the order of $10^{4}$ or higher which prevents the fountain scheme from efficiently supporting the real-time applications (i.e. low delay) [10]. In [11], the authors have shown that the small block size (i.e. $K=500$ ) of LT codes with small $\varepsilon$ (i.e. $3 \%$ ) can be achieved by using message-passing and Gaussian elimination together for decoding. Gaussian elimination is applied after the messagepassing algorithm. Combining both methods for decoding can have lower overhead and higher computation complexity in comparison with only using the message-passing algorithm to decode. For $K=500$, the complexity of using both methods is around of $25 \%$ of the complexity of only using messagepassing decoding, but the overhead of using both methods can be reduced from $42 \%$ to $3 \%$ [11]. In this paper, we combine both methods to decode LT codes.

Fountain codes are designed for communication over Erasure Channels, which means that the encoded packet is either received error free or not received at all. However, the wireless channels are not erasure, but fading and noisy channels. In practical systems, fountain codes are used in combination with other error correction algorithms to convert the noisy channels into erasure channels, often Low-Density ParityCheck (LDPC) codes [9]. In this paper, LDPC codes together 
with Cyclic Redundancy Check (CRC) are employed to convert the channel.

Our FEC encoding scheme is performed in the following order: $K$ source packets are encoded by fountain codes first. To each fountain-encoded packet, a CRC is first added and the packet is encoded by a LDPC code. So, the source data is first encoded jointly over all the sub-carriers by fountain codes, then encoded separately over one sub-carrier by LDPC plus CRC codes.

At the receiver, each fountain-encoded packet is first LDPC decoded when the SNR is equal to or higher than the threshold (i.e. BER $\leq 10^{-5}$ ). The received packet is discarded if its energy is below the threshold. If the LDPC decoding fails, the received packet is discarded as well. If the LDPC decoding succeeds, the CRC is used to identify the undetected error from LDPC codes. If the CRC decoder fails, the receiver also assumes that the whole packet has been lost. Once the receiver has collected $N$ surviving fountain-encoded packets, it starts to recover the source data.

\section{SySTEM MODEL}

The opportunistic error correction layer is based on fountain codes which have been explained in the above sections. This proposed cross layer can be applied in the OFDM systems. In this paper, the IEEE 802.11a system is taken as an example of OFDM systems.

The FEC layer in the current IEEE 802.11a system is based on Rate Compatible Punctured Codes (RCPC) [12]. Although this solution works well in practical systems, it is not optimal. First, because packets that have encountered a low-energy channel are still processed by the decoders. This is a waste of processing power. In addition, this FEC layer is based on worst case scenarios. However, worst case conditions do not happen all the time. This means that for most packets, the code rate and hence capacity can be increased.

In Figure 2, the proposed new error correction scheme is depicted. The key idea is to generate additional packets by the fountain encoder. First, the source packets are encoded by the fountain encoder. Then, a CRC checksum is added to each fountain-encoded packet and LDPC encoding is applied. On each sub-carrier, a fountain-encoded packet is transmitted. Thus, multiple packets are transmitted simultaneously, using frequency division multiplexing.

At the receiver side, we assume that the synchronization and channel estimation are perfect. If the SNR of the sub-carrier is equal to or above the threshold, the received fountain-encoded packet will go through LDPC decoding, otherwise it will be discarded. This means that the receiver is allowed to discard low-energy sub-carriers (i.e. packets) to lower the processing power consumption. After the LDPC decoding, the CRC checksum is used to discard the erroneous packets. As only packets with a high SNR are processed by the receiver, this will not happen often. When the receiver has collected enough fountain-encoded packets, it starts to recover the source data.

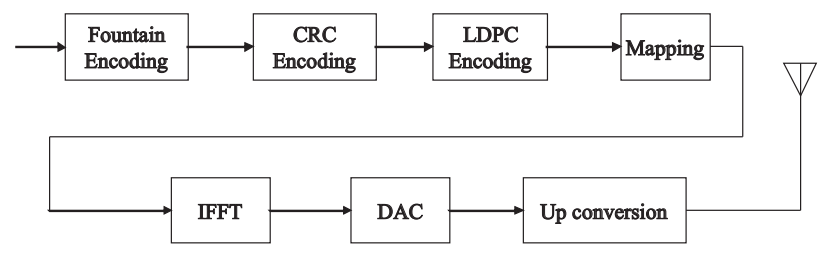

(a) Transmitter

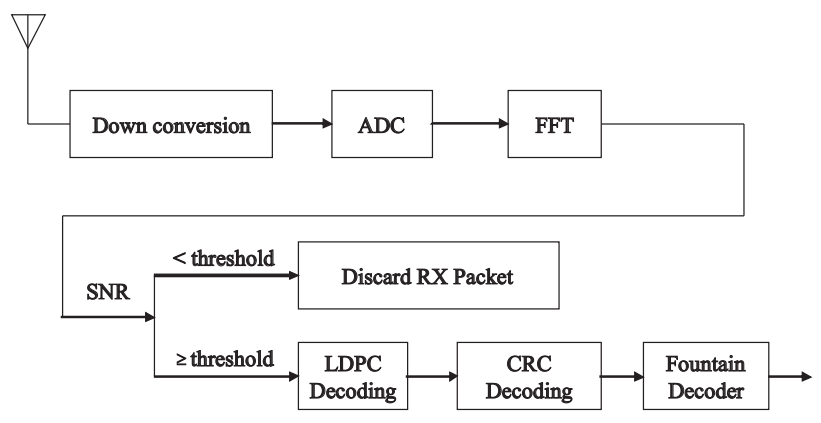

(b) Receiver

Fig. 2. Proposed IEEE 802.11a transmitter (top) and receiver (bottom).

\section{Performance Analysis}

In this section, we analyze the performance of our proposed opportunistic error correction layer. We compare four FEC schemes by simulation as follows:

- FEC I: RCPC with $R=0.5$ from the IEEE 802.11a standard [12].

- FEC II: LDPC codes with $R=0.5$ from the IEEE 802.11n standard [13] ( $\mathrm{n}=648)$.

- FEC III: fountain codes with the $(175,255)$ LDPC code plus 7-bit CRC using the transmission Mode I, which is the opportunistic error correction layer.

- FEC IV: fountain codes with the $(175,255)$ LDPC codes plus 7-bit CRC using the transmission Mode II.

In the simulation, we transmit 8000 blocks of source packets over Channel model A. Each block consists of 590 source packets with a length of 168 bits. With the same code rate of $R=0.5$, the source packets are encoded by FEC I, II, III and IV, respectively. Afterwards, they are mapped into QAM-16 symbols before the OFDM modulation.

For the case of FEC III and IV, each burst is encoded by a LT code (with parameters $c=0.03, \sigma=0.3$ ) and decoded by the message-passing algorithm and Gaussian elimination together. From [11], we know that 3\% overhead is required to recover the source packets successfully. To each fountainencoded packet, a 7-bit CRC is added, then the $(175,255)$ LDPC encoder is applied. Under the condition of the same code rate (i.e. $R=0.5$ ), we are allowed to discard $21 \%^{1}$ of transmitted packets. In FEC III, we transmit one packet per sub-carrier. In this case, we are allowed to discard 10 sub-carriers (i.e $21 \%$ of 48 data sub-carriers). In FEC IV, we

${ }^{1} 21 \% \approx 1-\frac{R}{R_{1} \times R_{2}}$, where $R$ is the effective code rate (i.e. 0.5 ), $R_{1}$ is the code rate of LT codes (i.e. $\frac{1}{1.03} \approx 0.97$ ) and $R_{2}$ is the code rate of the $(175,255)$ LDPC code with 7-bit CRC (i.e. $\frac{168}{255} \approx 0.66$ ) 


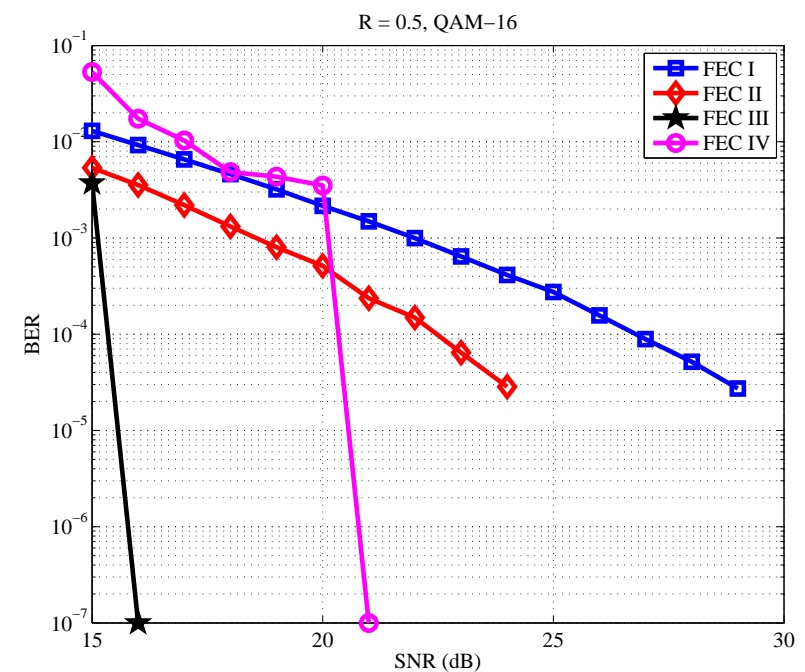

Fig. 3. Performance comparison between FEC I, II, III and IV at R = 0.5 (without interleaving in FEC I and II). For SNR $=16 \mathrm{~dB}$ or higher, no errors are detected in FEC III. So, for $\mathrm{SNR}=16 \mathrm{~dB}$, we represent $\mathrm{BER}=0$ by $10^{-7}$. Same for FEC IV.

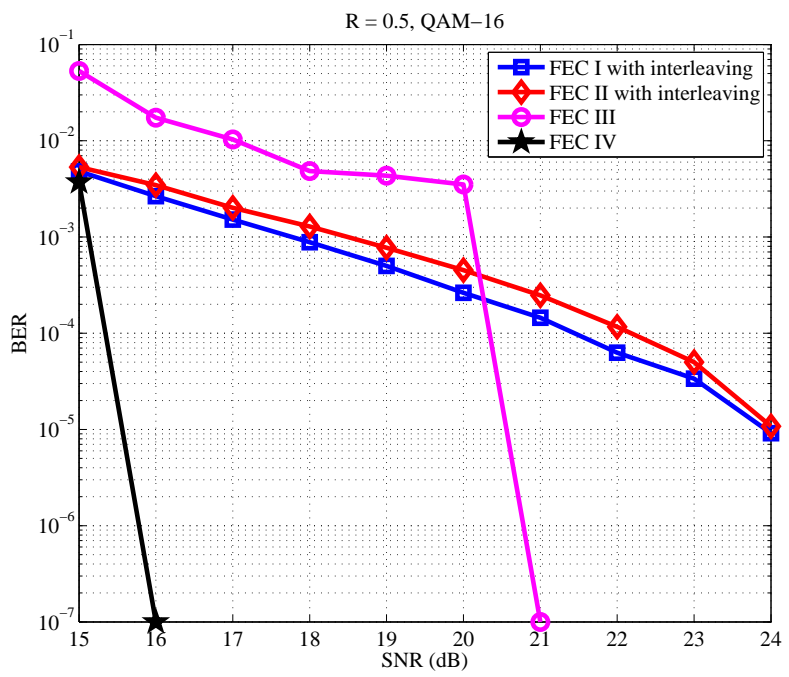

Fig. 4. Performance comparison between FEC I, II, III and IV at R = 0.5 (with interleaving in FEC I and II). For SNR $=16 \mathrm{~dB}$ or higher, no errors are detected in FEC III. So, for SNR $=16 \mathrm{~dB}$, we represent BER $=0$ by $10^{-7}$. Same for FEC IV.

transmit each fountain-encoded packet over all the data subcarriers. Same as FEC III, we are allowed to have $21 \%$ packet loss in FEC IV.

Figure 3 shows the simulation results with perfect channel knowledge. From this figure, we can see the followings:

- FEC I has a BER of $10^{-5}$ or lower when $\mathrm{SNR} \geq 29 \mathrm{~dB}$.

- FEC II has a BER of $10^{-5}$ or lower when $\mathrm{SNR} \geq 24 \mathrm{~dB}$.

- FEC III is error free when $\mathrm{SNR} \geq 16 \mathrm{~dB}$.

- FEC IV is error free when $\mathrm{SNR} \geq 21 \mathrm{~dB}$.

So, FEC III has a gain of around $13.5 \mathrm{~dB}$ comparing with FEC I, $8.5 \mathrm{~dB}$ comparing to FEC II and $5 \mathrm{~dB}$ comparing to
FEC IV.

As we know, RCPC has a good performance for random bit errors. Interleaving is employed to mitigate burst errors. To improve the performance of FEC I, an interleaver is added before the RCPC encoder. Also, an interleaver is inserted in FEC II to check whether interleaver can increase the performance of LDPC codes. Figure 4 shows the simulation results. Both FEC I and II with interleaving have a BER of $10^{-5}$ or lower when $\mathrm{SNR} \geq 24 \mathrm{~dB}$. In this case, FEC III has a gain of $8.5 \mathrm{~dB}$ comparing to FEC I and II. In addition, interleaving makes FEC I behave slightly better than FEC II and gives a gain of $5 \mathrm{~dB}$ to FEC I but no gain to FEC II.

From Figure 3 and 4, we can see that FEC III works better than the other FEC schemes. FEC III has a different transmission mode than the others. In FEC III, each packet is transmitted over one sub-carrier. In the OFDM system, each sub-carrier can be modeled as a flat fading channel. With the channel knowledge, the receiver knows how many sub-carriers (i.e. packets) will be lost. Actually, the surviving sub-carriers have a higher SNR than the threshold of the used LDPC codes, which means that the channel capacity of the surviving sub-carriers can not be fully utilized. But if one fountainencoded packet is transmitted over all the sub-carriers, the subcarrier with high energy can compensate for the subcarrier with deep fading. In this sense, transmission Mode II should have a smaller error probability than Mode I for the same code rate, which is stated in [3]. In order to show this, we compare FEC III and FEC IV without using fountain codes in the same simulation condition as Figure 3 and 4. Figure 5 shows the BER performance of both transmission modes. We can see that the transmission Mode II has a lower BER than the transmission Mode $\mathrm{I}$ at the same code rate when $\mathrm{SNR}=15 \sim 16 \mathrm{~dB}$.

However, Figure 3 and 4 do not show the same result as Figure 5. In Figure 3 and 4, FEC III has a lower BER than FEC IV at SNR $=15 \mathrm{~dB}$. When SNR $=16 \mathrm{~dB}$, FEC III is error free but FEC IV still has a BER of around $10^{-2}$. By using fountain codes, the receiver does not care about the total number of errors in the received packets but only cares about the total number of the error-free packets. Figure 6 shows the outage probability $\mathrm{P}_{\text {out }}$ of the packet loss $\epsilon$ :

$$
\mathrm{P}_{\text {out }}=\mathbb{P}\{\text { the percentage of packet loss }<\epsilon\}
$$

In this figure, we can see that FEC III has a lower packet loss than FEC IV when we allow more than $7 \%$ packet loss. Therefore, from Figure 5 and Figure 6 we conclude that the transmission Mode I has a larger BER comparing transmission mode II but the errors exist in less number of packets than the transmission mode II when the packet loss is larger than $7 \%$. As mentioned earlier, we allow $21 \%$ packet loss in FEC III and IV in order to have the same code rate of FEC I and II. Fountain codes can recover the source data once the receiver has collected enough error-free fountain-encoded packets. That explains why FEC III works better than FEC IV. Also, because of this and the characteristics of fountain codes, FEC III works better than FEC I and FEC II. 


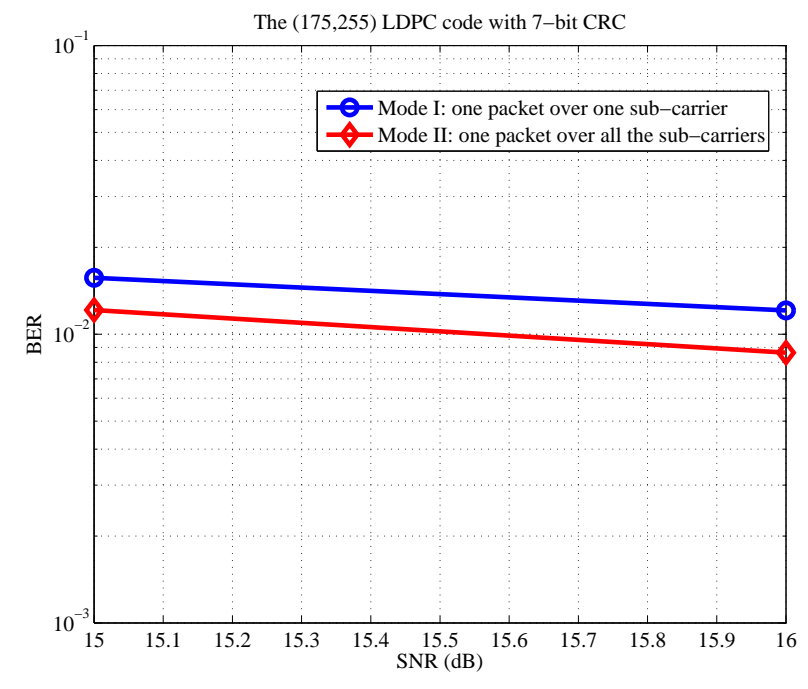

Fig. 5. Comparison in BER between the transmission Mode I and Mode II using the $(175,255)$ LDPC code with 7-bit CRC.

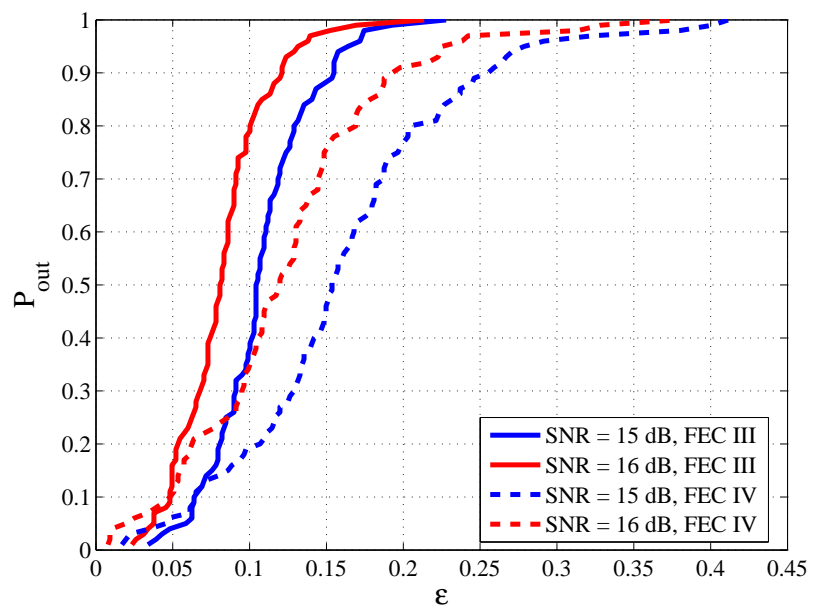

Fig. 6. Comparison in the outage probability of the packet loss between the transmission Mode I and Mode II using the $(175,255)$ LDPC code with 7-bit CRC.

\section{CONCLUSIONS}

In this paper, we propose a novel cross-coding approach based on fountain codes for OFDM systems, which combines the separate coding and the joint coding over all the subcarriers. It is called the opportunistic error correction layer, because it does not process all the received packets but only the packets from high-energy channels. In such a case, the processing power consumption can be reduced correspondingly. This new method has a gain of $8.5 \mathrm{~dB}$ in comparison with the current FEC layer used in the IEEE 802.11a system and the LDPC codes with interleaving used in the IEEE 802.11n system. Also, it has a gain of $5 \mathrm{~dB}$ comparing with the case that each fountain-encoded packet is transmitted over all the sub-carriers.

\section{ACKNOWLEDGEMENTS}

We thank the Dutch Ministry of Economic Affairs under the IOP Generic Communication - SenterNovem Program for the financial support.

\section{REFERENCES}

[1] A. Bahai, B. Saltzberg, and M. Ergen, Multi-carrier Digital Communications: Theory and Applications of OFDM. Springer, 2004.

[2] A. Carlson, Communication Systems. McGraw-Hill New York etc., 1975.

[3] D. Tse and P. Viswanath, Fundamentals of Wireless Communication. Cambridge University Press, 2005.

[4] D. J. C. MacKay, "Fountain Codes," IEE Communications, vol. 152, no. 6, pp. 1062-1068, 2005.

[5] European Telecom Standards Institue, "Channel Models for HIPERLAN/2 in Different Indoor Scenarios," 1998.

[6] Y. Kou, S. Lin, and M. P. C. Fossorier, "Low-Density Parity-Check Codes Based on Finite Geometries: A Rediscovery and New Results," IEEE Transactions on Information Theory, vol. 47, no. 7, pp. 27112736, 2001.

[7] M. Luby, "LT Codes," Proceedings of the 43rd Annual IEEE Symposium on Foundations of Computer Science, pp. 271-282, 2002.

[8] A. Shokrollahi, "Raptor Codes," IEEE Transaction on Information Theory, vol. 52, 2006.

[9] D.J.C. MacKay, Information Theory, Interference, and Learning Algorithms. Cambridge University Press, 2003.

[10] H. Zhu, C. Zhang, and J. Lu, "Designing of Fountain Codes with Short Code-Length," The 3rd International Workshop on Signal Design and Its Applications in Communications (IWSDA), pp. 65-68, 2007.

[11] X. Shao, R. Schiphorst, and C. H. Slump, "An Opportunistic Error Correction Layer for OFDM Systems," EURASIP Journal on Wireless Communications and Networking, 2009.

[12] IEEE, "Wireless LAN Medium Access Control (MAC) and Physical Layer (PHY) Specifications, High-Speed Pysical Layer in the $5 \mathrm{GHz}$ Band (IEEE 802.11a Standard, Part 11)," 1999.

[13] IEEE, "Draft Standards for Wireless LAN Medium Access Control (MAC) and Physical Layer (PHY) Specifications, Enhancements for Higher Throughput (IEEE 802.11n Standard, Part 11),” Jan, 2007. 